\title{
Prenatal sensitization of a postnatal trigger for metabolic disease
}

\author{
Susan K. Murphy \\ Department of Obstetrics and Gynecology, Division of Gynecologic Oncology, Duke University Medical Center, Durham, North Carolina, USA.
}

\begin{abstract}
Intrahepatic cholestasis of pregnancy (ICP), marked by elevated maternal serum bile acid levels, occurs in late pregnancy and is often associated with poor perinatal outcomes. In this issue of the JCI, Papacleovoulou et al. analyze the long-term consequences of ICP and find that teens born to mothers with ICP exhibit enhanced characteristics of metabolic syndrome relative to controls. The authors also used a new ICP mouse model to support and extend these findings, demonstrating that in utero exposure to bile acids induces persistent epigenetic alterations and abnormal placental lipogenesis, setting the stage for later metabolic dysfunction.
\end{abstract}

ICP is an uncomfortable condition of late pregnancy characterized by elevated levels of bile in the maternal serum and abnormal serum liver tests. The etiology of ICP is largely unknown. However, the risk for developing ICP differs by geography, with higher rates reported in Chile and Scandinavia (1), although its prevalence has decreased over the last few decades to about $2 \%$ of all pregnancies in these countries (2). An increased risk of ICP is also positively associated with parity, carrying multiples, maternal age over 35 years, and a family history of biliary disease (3). The major symptom is intense itching, which is often relieved when the mother is treated with ursodeoxycholic acid (UDCA) (4). While the discomfort resolves for the mother shortly after birth, there can be complications for the infant, including preterm birth, exposure to meconium in the amniotic fluid, and increased risk of fetal death in late pregnancy. Early delivery is aimed at reducing these complications.

Disruption of bile acid homeostasis resulting in cholestasis during pregnancy is thought to be triggered by the hormonal milieu present (5). The occurrence of familial cases and the identification of specific mutations in bile transporter genes (6) support the hypothesis that genetic variants may predispose women to the condition. Outcomes have been studied primarily in the mother immediately postpartum and at parturition for the newborn (7), and little attention has been given to the long-term

Conflict of interest: The author has declared that no conflict of interest exists.

Citation for this article: J Clin Invest. 2013; 123(7):2786-2788. doi:10.1172/JCI69399. ramifications for the child who was exposed to high levels of bile acids during gestation.

\section{Long-term consequences}

In this issue of the JCI, Papacleovoulou et al. (8) provide the first evidence that ICP quences in the child. The authors studied data obtained over a 16-year period from nondiabetic mothers and children who were part of a large birth cohort in northern Finland. There were 45 cholestatic pregnancies among 7,853 births during 1984-1985, and these women were not treated with UDCA, so the developing fetus was fully exposed to the effects of the elevated bile acids. The mothers' BMI did not differ between the ICP and normal pregnancies. Compared with teens born to mothers with normal pregnancies, both male and female teens born to mothers with ICP showed significantly increased BMI. Males had elevated fasting insulin levels approaching insulin resistance and elevated apolipoprotein B and LDL cholesterol, while females showed increased hip girth and lower HDL cholesterol. These changes were directionally and ominously shifted toward values observed in metabolic syndrome.

To further understand the mechanisms involved, the authors developed a mouse model of hypercholanemia. Prior to and throughout gestation, WT female mice were fed a normal diet or one enriched with cholic acid, the major bile acid produced in the liver from cholesterol. The cholic acid-supplemented females had elevated serum and liver bile acid levels, consistent with a cholestatic phenotype. At delivery, all mothers were fed a normal diet. The pups were weaned at is associated with long-term health conse-
3 weeks, and at 12 weeks of age were divided into groups fed either a normal chow or a Western diet. Papacleovoulou et al. (8) demonstrated that the accelerated weight gain associated with a Western diet was markedly exacerbated by exposure to cholic acid during gestation in female mice. Furthermore, the cholic acid-exposed, Western diet-fed female mice had a more severe obesogenic and diabetic phenotype than females born to cholestatic mothers but then fed a normal diet. Gene expression profiles obtained from the liver and white adipose tissues of these mice also showed an increased inflammatory response (8).

The phenotype of the cholic acid-exposed, Western diet-fed mice is perhaps expected, but the more remarkable findings of these experiments came from studying the cholic acid-exposed mice fed a normal diet compared with the controls. Gestational exposure to cholic acid followed by consumption of a normal diet was associated with increased FFAs in the liver, mild hepatosteatosis, and altered serum inflammatory cytokine profiles in addition to increased adipocytokine levels consistent with insulin resistance. Transcriptome profiling indicated enrichment of the genes involved in inflammatory, immunological, cardiovascular, and metabolic disorders. The implication of these findings is that these mice were primed during gestation to develop metabolic disorders, and their progression to that state depended on their later dietary intake (8).

\section{Gestational conditions setting the stage for obesity?}

The early origins of disease hypothesis postulates that the risk of developing particular chronic diseases later in life, including cardiovascular disease, metabolic disorders, obesity, and diabetes, is directly associated with the environmental conditions encountered during early life (9). This hypothesis emerged from observations of groups of individuals who, as a population and for a discrete period of time, endured nutritional deprivation. Some of these individuals were 


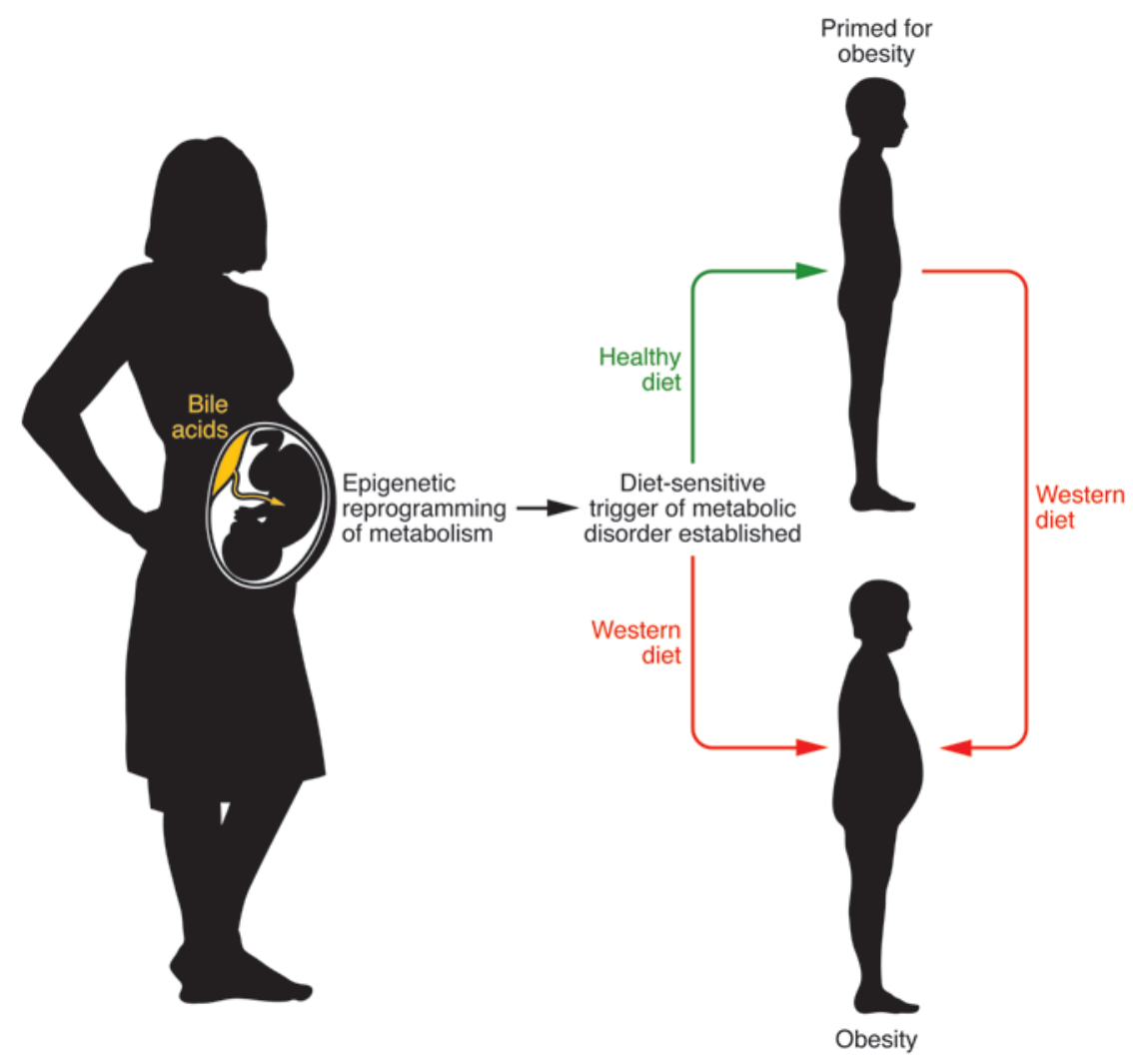

Figure 1

Exposure to bile acids in utero leads to metabolic profile reprogramming in the developing child, likely through epigenetic alterations. This reprogramming establishes a diet-sensitive trigger that responds to poor dietary choice by increasing the likelihood of developing metabolic syndrome. The manifestation of this syndrome may be constrained by consumption of a healthy diet.

pregnant at the time of deprivation, and health assessments of the offspring of the nutritionally deprived mothers have shown that severe caloric restriction experienced in utero increases the risk of disease in adulthood (10). The findings presented by Papacleovoulou et al. (8) that gestational exposure to cholestasis programs a metabolic profile with a trajectory toward obesity and diabetes and a trigger sensitive to subsequent diet are consistent with this hypothesis. Epigenetic changes are thought to play a central role in affecting these outcomes (11), and indeed, DNA methylation changes resulting from the maternal diet have already been shown to affect an obese phenotype in humans and animal models $(12,13)$.

To address the potential role of epigenetics in mediating the effects of gestational cholic acid exposure, Papacleovoulou et al. (8) used the viable yellow agouti $\left(A^{v y}\right)$ mouse model as an epigenetic "biosensor" (14). The agouti gene product causes hair follicle melanocytes to produce either black eumelanin (a) or yellow phaeomelanin $(A)$ in a temporally depen- dent fashion during the hair growth cycle. Normally, this results in the production of a short subapical band of yellow color on an otherwise black hair shaft, giving the mouse its brownish coat color. The $A^{v y}$ mouse harbors an IAP retrotransposon insertion upstream of agouti, which contains a cryptic promoter that is constitutively active when unmethylated. This leads to the production of read-through transcripts containing agouti and to a yellow coat color, with the extent of yellow coloration dependent on the degree of methylation present. In their study, Papacleovoulou and colleagues (8) showed that mother mice fed a cholic acidenriched diet prior to and throughout gestation produced pups with a shifted coat color distribution relative to pups born to mother mice fed a normal chow diet. Methylation levels measured at the cryptic IAP promoter were consistent with those expected for the coat colors observed in a representative sample of the pups. Curiously, exposure to the maternal cholic acid-enriched diet differentially skewed the distribution of coat color in the male and female pups. While the exposed males showed a shift toward more yellow coloration (presumed decrease in methylation), the exposed females showed a shift toward more brown coat coloration (presumed increase in methylation). Sexspecific epigenetic effects following in utero exposures have been reported elsewhere (15), but the reasons for these differences are not yet clear. The conclusion to be drawn from this experiment is that prenatal cholic acid exposure can elicit changes in DNA methylation. The relatively small sample size and lack of methylation analysis across the coat color distributions in the exposed and control pups are limitations of the work. Importantly, Papacleovoulou et al. (8) did not determine whether changes in methylation occur at other loci directly relevant to the observed phenotypes from in utero cholic acid exposure.

Changes in placental function in response to a cholic acid-enriched diet were also revealed in the study. Papacleovoulou and colleagues (8) found that the placentas from the cholestatic mouse mothers showed altered lipid synthesis and storage, and the exposed fetuses also exhibited a cholestatic phenotype. Increased cholesterol was transported across the placenta into the fetuses, and the fetal livers were undergoing de novo synthesis of cholesterol and fatty acids. Similarly, human placentas from ICPs showed increased lipid synthesis and storage as well as enhanced cholesterol efflux measured in cultured cytotrophoblasts (8).

\section{Conclusions and remaining questions}

This is the first demonstration that cholestasis of pregnancy results in an altered metabolic profile in the child, an effect that persists for over a decade. The results suggest that in utero exposure to bile acids results in a reprogramming of metabolism that is fully realized under conditions of suboptimal nutritional intake, which may or may not occur later in life, depending on behavior (Figure 1). Still, questions remain. Treatment with UCDA reduced bile acid levels in women with cholestasis; can it also prevent the longterm metabolic consequences in the child? Although cholestasis is usually not symptomatic until the second or third trimester, is it present earlier in a latent form, and if so, at what point should UCDA be administered to provide maximum benefit? Can the effects of in utero exposure to cholestasis or even to subacute cholestasis partially explain the rising levels of obesity in children? If so, 
can this trajectory be modified using UCDA? The influence of cholic acid on DNA methylation in the agouti mouse model very likely occurred during embryogenesis, since all germ layers showed equivalent changes in methylation. If ICP is truly specific to the second or third trimester, the effects in the agouti model may be very different from any epigenomic effects elicited by ICP. The mouse model of cholestasis will be useful in future work to more fully characterize these epigenomic effects and to identify the particularly relevant windows of exposure.

Understanding the roots of metabolic dysfunction may provide inroads to improved treatment options involving the specific genes whose expression is altered by cholestatic programming. Obesity has major ramifications for overall health and well-being and is at epidemic proportions in the United States. This cholestatic mouse model may also provide insights that are generalizable to other factors that predispose to metabolic dysfunction.

\section{Acknowledgments}

The author's research is supported by NIH grants AA019399, ES016772, and DK085173.

Address correspondence to: Susan K. Murphy, Duke University Medical Center, 226 B Wing, Levine Science Research Center, Box 91012, Research Drive, Durham, North Carolina 27708, USA. Phone: 919.681.3423; Fax: 919.684.5336; E-mail: susan.murphy@duke.edu.

1. Geenes V, Williamson C. Intrahepatic cholestasis of pregnancy. World J Gastroenterol. 2009; 15(17):2049-2066

2. Pusl T, Beuers U. Intrahepatic cholestasis of pregnancy. Orphanet J Rare Dis. 2007;2:26.

3. Pathak B, Sheibani L, Lee RH. Cholestasis of pregnancy. Obstet Gynecol Clin North Am. 2010;37(2):269-282.

4. Beuers U. Drug insight: Mechanisms and sites of action of ursodeoxycholic acid in cholestasis. Nat Clin Pract Gastroenterol Hepatol. 2006;3(6):318-328.

5. Joshi D, James A, Quaglia A, Westbrook RH, Heneghan MA. Liver disease in pregnancy. Lancet. 2010;375(9714):594-605.

6. Pauli-Magnus C, Meier PJ, Stieger B. Genetic determinants of drug-induced cholestasis and intra- hepatic cholestasis of pregnancy. Semin Liver Dis. 2010;30(2):147-159.

7. Wikström Shemer E, Marschall H, Ludvigsson J, Stephansson O. Intrahepatic cholestasis of pregnancy and associated adverse pregnancy and fetal outcomes: a 12-year population-based cohort study. BJOG. 2013;120(6):717-723

8. Papacleovoulou G, et al. Maternal cholestasis during pregnancy programs metabolic disease in offspring. J Clin Invest. 2013;123(7):3172-3181

9. Barker DJ. The fetal and infant origins of adult disease. BMJ. 1990;301(6761):1111.

10. Roseboom T, de Rooij S, Painter R. The Dutch famine and its long-term consequences for adult health. Early Hum Dev. 2006;82(8):485-491.

11. Hochberg Z, et al. Child health, developmental plasticity, and epigenetic programming. Endocr Rev. 2011;32(2):159-224

12. Godfrey KM, et al. Epigenetic gene promoter methylation at birth is associated with child's later adiposity. Diabetes. 2011;60(5):1528-1534.

13. Lillycrop KA, Burdge GC. Epigenetic changes in early life and future risk of obesity. Int J Obes (Lond). 2011;35(1):72-83.

14. Dolinoy DC. The agouti mouse model: an epigenetic biosensor for nutritional and environmental alterations on the fetal epigenome. Nutr Rev. 2008;66(suppl 1):S7-S11.

15. Gabory A, Attig L, Junien C. Sexual dimorphism in environmental epigenetic programming. Mol Cell Endocrinol. 2009;304(1-2):8-18.

\title{
Mitochondrial TCA cycle intermediates regulate body fluid and acid-base balance
}

\author{
János Peti-Peterdi
}

Department of Physiology and Biophysics and Department of Medicine, Zilkha Neurogenetic Institute, University of Southern California, Los Angeles, California, USA.

\begin{abstract}
Intrarenal control mechanisms play an important role in the maintenance of body fluid and electrolyte balance and $\mathrm{pH}$ homeostasis. Recent discoveries of new ion transport and regulatory pathways in the distal nephron and collecting duct system have helped to better our understanding of these critical kidney functions and identified new potential therapeutic targets and approaches. In this issue of the JCI, Tokonami et al. report on the function of an exciting new paracrine mediator, the mitochondrial the citric acid (TCA) cycle intermediate $\alpha$-ketoglutarate ( $\alpha \mathrm{KG}$ ), which via its OXGR1 receptor plays an unexpected, nontraditional role in the adaptive regulation of renal $\mathrm{HCO}_{3}{ }^{-}$secretion and salt reabsorption.
\end{abstract}

The distal nephron and collecting ducts are the final segments of the renal tubular network, which enable fine-tuning of systemic $\mathrm{NaCl}$, water, $\mathrm{K}^{+}$, and acid-base balance. According to the classical view, the principal cells respond to systemic hormones like aldosterone and vasopressin to modulate transport of salt, water, and $\mathrm{K}^{+}$, while interca-

Conflict of interest: The author received research support from Boehringer Ingelheim.

Citation for this article: J Clin Invest. 2013; 123(7):2788-2790. doi:10.1172/JCI68095. lated cells can excrete either $\mathrm{H}^{+}$(type A cells) or $\mathrm{HCO}_{3}{ }^{-}$(type $\mathrm{B}$ cells), depending on the body's acid-base status (1). Type B intercalated cells extrude $\mathrm{H}^{+}$through a basolateral vacuolar $\mathrm{H}^{+}$-ATPase and secrete $\mathrm{HCO}_{3}{ }^{-}$into urine via the apical $\mathrm{Cl}^{-} / \mathrm{HCO}_{3}{ }^{-}$exchanger pendrin (1). Another intercalated cell subtype, non-A-non-B cells, expresses both the $\mathrm{H}^{+}$pump and pendrin at the apical membrane (2). These intercalated cell types and transport mechanisms play an important role in maintaining the normal plasma $\mathrm{pH}$ of 7.4 (acid-base balance) via their corrective response to an acid or alkali load. For example, increased dietary acid intake (an example of metabolic acidosis) causes a reversal of polarity of $\mathrm{HCO}_{3}{ }^{-}$flux in the distal nephron and collecting duct, as $\mathrm{HCO}_{3}{ }^{-}$-secreting type $\mathrm{B}$ intercalated cells are remodeled to become $\mathrm{H}^{+}$-secreting type A intercalated cells (3). Conversely, dietary alkali load (an example of metabolic alkalosis) triggers an adaptive response by intercalated cells in which their population density is shifted to the $\mathrm{B}$ ( $\mathrm{HCO}_{3}^{-}$-secreting) type (4).

However, in addition to their apparent role in $\mathrm{pH}$ balance, a new paradigm of the distal nephron and collecting duct is currently emerging in which, via newly identified functions of intercalated cells and paracrine mechanisms, this structure plays a critical role in renal physiology, body electrolyte and water homeostasis, and blood pressure control (5). For example, the Eladari and Chambrey groups recently demonstrated the expression of the $\mathrm{Na}^{+}$-driven $\mathrm{Cl}^{-} / \mathrm{HCO}_{3}{ }^{-}$exchanger $\mathrm{NDCBE}(\mathrm{Slc} 4 \mathrm{a} 8)$ in 